\title{
The Importance of Iron Administration in Correcting Anaemia after Major Surgery
}

\author{
Mirela Tiglis,2, Cristian Cobilinschi1,2, Liliana Elena Mirea1,2, Alexandru Emil Băetu1,2, \\ Ileana Peride ${ }^{1}$, Tiberiu Paul Neagu1 ${ }^{*}$, Andrei Niculae ${ }^{1}$, Ionel Alexandru Checherită ${ }^{1}$, loana \\ Marina Grintescu1,2 \\ 1 "Carol Davila" University of Medicine and Pharmacy, Bucharest, Romania \\ 2 Emergency Clinical Hospital of Bucharest, Romania
}

\begin{abstract}
Introduction: Postoperative anaemia can affect more than $90 \%$ of patients undergoing major surgeries. Patients develop an absolute iron deficiency in the face of significant blood loss or preoperative anaemia and major surgery. Studies have shown the negative impact of these factors on transfusion requirements, infections, increased hospitalisation and long-term morbidities. Aim of the study: The research was performed to determine the correlation between intravenous iron administration in the postoperative period and improved haemoglobin correction trend. Material and methods: A prospective study was conducted to screen and treat iron deficiency in patients undergoing major surgery associated with significant bleeding. For iron deficiency anaemia screening, in the postoperative period, the following bioumoral parameters were assessed: haemoglobin, serum iron, transferrin saturation (TSAT), and ferritin, direct serum total iron-binding capacity (dTIBC), mean corpuscular volume (MCV) and mean corpuscular haemoglobin $(\mathrm{MCH})$. In addition, serum glucose, fibrinogen, urea, creatinine and lactate values were also collected. Results: Twenty-one patients undergoing major surgeries (52,38\% were emergency and $47,61 \%$ elective interventions) were included in the study. Iron deficiency, as defined by ferritin 100-300 $\mu \mathrm{g} / \mathrm{L}$ along with transferrin saturation (TSAT) $<20 \%$, mean corpuscular volume $(\mathrm{MVC})<92 \mathrm{fL}$, mean corpuscular haemoglobin $(\mathrm{MCH})<33 \mathrm{~g} / \mathrm{dL}$, serum iron $<10 \mu \mathrm{mol} / \mathrm{L}$ and direct serum total iron-binding capacity (dTIBC) $>36 \mu \mathrm{mol} / \mathrm{L}$, was identified in all cases. To correct the deficit and optimise the haematological status, all patients received intravenous ferric carboxymaltose (500-1000 $\mathrm{mg}$, single dose). Using Quadratic statistical analysis, the trend of haemoglobin correction was found to be a favourable one. Conclusion: The administration of intravenous ferric carboxymaltose in the postoperative period showed the beneficial effect of this type of intervention on the haemoglobin correction trend in these groups of patients.
\end{abstract}

Keywords: major surgery, anaemia, postoperative iron deficiency, intravenous iron therapy

Received: 30 June 2021 / Accepted: 21 July 2021

\section{BACKGROUND}

World Health Organization (WHO) defines anaemia as a haemoglobin level under $13 \mathrm{~g} / \mathrm{dL}$ in men and under $12 \mathrm{~g} / \mathrm{dL}$ in women, affecting more than $30 \%$ of the worldwide population [1]. In surgical patients, preoperative anaemia has a prevalence of approximately $40 \%$ of patients, reaching $90 \%$ after major surgeries [2-4]. Iron deficiency anaemia (IDA) presents a high prevalence (affecting 1.2 billion people), and iron deficiency without anaemia (IDWA) being even more frequent [5, 6].

In case of significant blood volume loss, due to major surgery or prolonged hospitalisation [7], anaemia can develop or worsen, and it is usually associated with absolute or functional iron deficiency (the erythropoietic response is increased and outstrips the actual iron supply) $[8,9]$.

In normal conditions, the body contains about 3-5 grams of iron, almost 2 grams incorporated into haemoglobin and mature erythrocytes, and with each millilitre of lost blood, the body loses approximately 0.5 milligrams (mg) of iron [10,11].

Over the last years, various studies have shown the negative impact of anaemia and iron deficiency in surgical patients, leading to increased red blood cells transfusion requirements, infections, slower recovery, ischemic complications (especially myocardial infarction), acute kidney injury [12], prolonged length of hospital stay, increased readmission, a more unsatisfac- 
tory perioperative outcome with increased mortality and morbidity [13-15]. Furthermore, acquired hospital anaemia, and in many cases, superimposed acquired iron deficiency, persists long after patients discharge, even beyond six months [16-18]. Therefore, correcting iron deficiency after surgery can help patients correct anaemia more rapidly, offering 'the fuel' for stress erythropoiesis (increasing iron bioavailability).

Various human and experimental studies have shown that iron deficiency is associated with dysregulations of glycaemia and lipids metabolism $[19,20]$ or that high glucose levels can lead to iron overload and increase oxidative stress [21]. In addition, iron deficiency, due to impairing the homeostasis of some mitochondrial enzymes, leads to inappropriate glycolysis with subsequent lactate accumulation [22].

The study aimed to determine the influence of intravenous iron administration in the postoperative period on improving haemoglobin correction trends. In addition, the influence of serum glycaemia and lactate on the haematological status of these patients was also evaluated.

\section{MATERIAL AND METHODS}

\section{Study variables}

A prospective-study was undertaken for the screening and treatment of iron deficiency in patients undergoing major surgery associated with significant bleeding, admitted to Emergency Clinical Hospital of Bucharest between $1^{\text {st }}$ May to $31^{\text {th }}$ August 2020.

All of the following criteria were required for the patient to be included in the study:

- age between 18 and 90 years

- patients undergoing major surgery

- perioperative bleeding $>500 \mathrm{~mL}$ (or $>250 \mathrm{~mL}$ in patients with at least moderate anaemia before surgery)

- postoperative anaemia

- postoperative iron deficiency screening.

Only one of the following criteria was required for the patient to be excluded:

- perioperative red blood cells transfusion

- sepsis or septic shock development

- iron allergy

- persistent inflammatory state
- chronic renal disease

- chronic inflammatory disease

- lack of haemoglobin level measurements after iron administration.

The Ethical Committee of the Emergency Clinical Hospital has approved this study (No. 10404/2020) as part of an ongoing research for a $\mathrm{PhD}$ thesis. All patients included in this analysis have received and signed an informed consent form.

\section{Measurements and intervention}

For iron deficiency anaemia screening, in the postoperative period, the following bioumoral parameters were used:

- haemoglobin (preoperative, intraoperative, preiron administration, 48 and 72 hours after intervention)

- serum iron

- transferrin saturation (TSAT)

- ferritin

- direct serum total iron-binding capacity (dTIBC)

- mean corpuscular volume (MCV)

- mean corpuscular haemoglobin (MCH)

Glucose, fibrinogen, urea, creatinine and serum lactate levels were also measured.

Ferritin and fibrinogen values were used to evaluate the inflammatory status.

Due to the short time before hospital admission and surgical intervention (the large percent of emergency surgeries or the need to minimise the hospitalisation in the COVID-19 pandemic period) and because of significant intraoperative bleeding, intervention of optimising the haematological status was performed only during the postoperative period. Therefore, there was no paraclinical follow-up appointments after hospital discharge.

Following the calculated iron deficit (Ganzoni equation - Figure 1 [23]), the intervention consisted in administering either 500 or $1000 \mathrm{mg}$ of intravenous ferric carboxymaltose (Ferinject ${ }^{\oplus}$, Vifor Pharma) single infusion, under permanent surveillance.

The agent choice was made on availability in the hospital.

The primary outcome was to restore iron stores to improve erythropoiesis in an attempt to rapidly correct haemoglobin levels. 
Total Iron Deficit $=$ Weight $\{\mathrm{kg}\} \times($ Target $\mathrm{Hb}-$ Actual $\mathrm{Hb})\{\mathrm{g} / \mathrm{I}\} \times 2.4+$ Iron Stores $\{\mathrm{mg}\}$

Fig. 1. Ganzoni equation

\section{Statistical analysis}

For statistical analysis and graphics generation, the MedCalc 19.2.4 program was used. For mean, median and standard deviation calculation for each parameter, the " $t$-test" (one-sample $t$-test for descriptive analysis) was used, and for the group comparison, the ANOVA test with Bonferroni correction, Shapiro-Wilk test of normality and Mann-Whitney test for independent variables were used.

The significance level was set at $\alpha=0.05$. A p-value $<0.05$ was considered statistical significant.

\section{RESULTS}

After applying the inclusion and exclusion criteria, from the thirty patients to whom parenteral iron therapy was administered and was initially considered eligible, twenty-one remained in the study with a mean age (SD) of 58.90 (19.06) years, $38 \%$ being females and $62 \%$ males (Figure 2).

Regarding the type of surgery, $52.58 \%$ were emergency surgeries, and $47.61 \%$ were elective. The mean duration for the surgical procedure in multiple trauma was 88 minutes.

Basic patients' characteristics are presented in Table 1 .
Haemoglobin values variation between postoperative, intraoperative and pre-iron administration times are presented in Figure 3.

When anaemia and iron screening analyses were performed in the postoperative period (maximum 24 hours after surgery), iron deficiency was identified in all included patients (Table 2). The cut-offs used for iron deficiency were ferritin 100-300 $\mu \mathrm{g} / \mathrm{L}$ along with transferrin saturation (TSAT) 20\%, MVC $<92 \mathrm{fL}$, $\mathrm{MCH}<33 \mathrm{~g} / \mathrm{dL}$, serum iron $<10 \mu \mathrm{mol} / \mathrm{L}$ and dTIBC $>36 \mu \mathrm{mol} / \mathrm{L}$. Thirteen patients received $1000 \mathrm{mg}$ and three patients $500 \mathrm{mg}$ of ferric carboxymaltose in the first 48 hours after surgery, one patient $500 \mathrm{mg}$ and one $1000 \mathrm{mg}$ after 3-5 days and three patients received 1000 $\mathrm{mg}$ after seven days. The timing of iron administration differed according to patient status (renal function, inflammatory state). There were reported no adverse events related to iron administration.

Other bioumoral markers were analysed at 48 and 72 hours after an intervention to evaluate their influence on iron status in these patients. The absence of an acute inflammatory state was validated by the low levels of ferritin and fibrinogen (Table 2 and 3 ).

In studied patients, glucose value was correlated with haemoglobin correction trend at both analysed postoperative periods $(\mathrm{p}<0.0001)$.

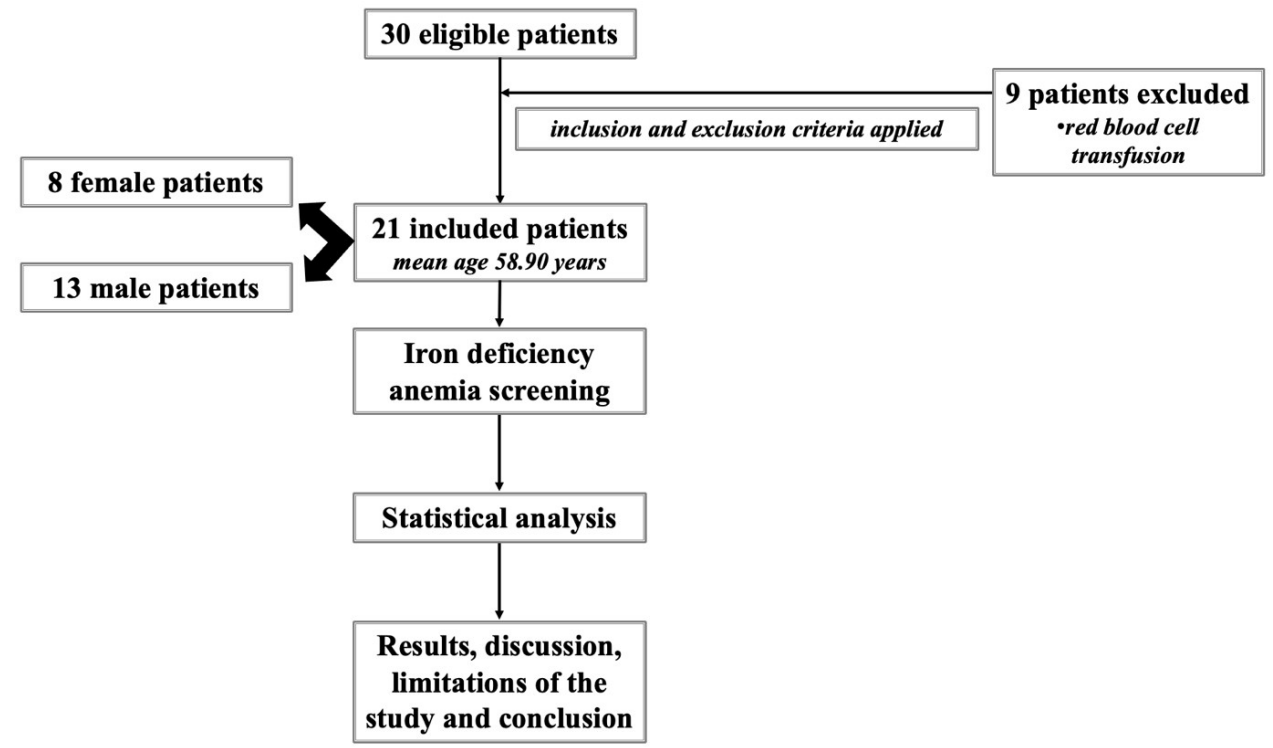

Fig. 2. Design of the study 
Table 1. Basic characteristics of included patients

\section{Characteristics}

Age in years. mean (SD)

Gender (number) (\%)

- female

- male

ASA* Score (number) (\%)

- ASA II

- ASA III

- ASA IV

Type of surgery (number) (\%)

- general surgery

- orthopaedics

- multiple trauma

Emergency vs elective (number) (\%)

- emergency

- elective

Surgery duration in minutes; mean (SD)

- general surgery

- orthopaedics

- multiple trauma

Intraoperative bleeding in $\mathrm{mL}$; mean (SD)

- general surgery

- orthopaedics

- multiple trauma

Haemoglobin variation in $\mathrm{g} / \mathrm{dL}$ (mean (SD)

- preoperative

- intraoperative

- pre-iron administration

- post-iron administration at 48 hours (I)

- post-iron administration at 72 hours (II)

Length of stay in hospital in days.

ASA = American Society of Anesthesiology, mL-milliliter, $\mathrm{mg}-$ milligram.

\section{Values}

19-86. mean 58.90(19.06)

8 (38.09)

$13(61.90)$

$4(19.05)$

14 (66.67)

$3(14.28)$

$7(33.33)$

9 (42.85)

$5(23.80)$

11 (52.38)

$10(47.61)$

172.85( 57.74)

155.62(41.18)

$88(41.66)$

745.71 (405.28)

653.7 (453.26)

610 (111.35)

$11.78(2.27)$

10.27 (1.81)

$8.51(0.95)$

$8.65(0.91)$

8.84( 0.86)

$5-23($ mean $=10)$

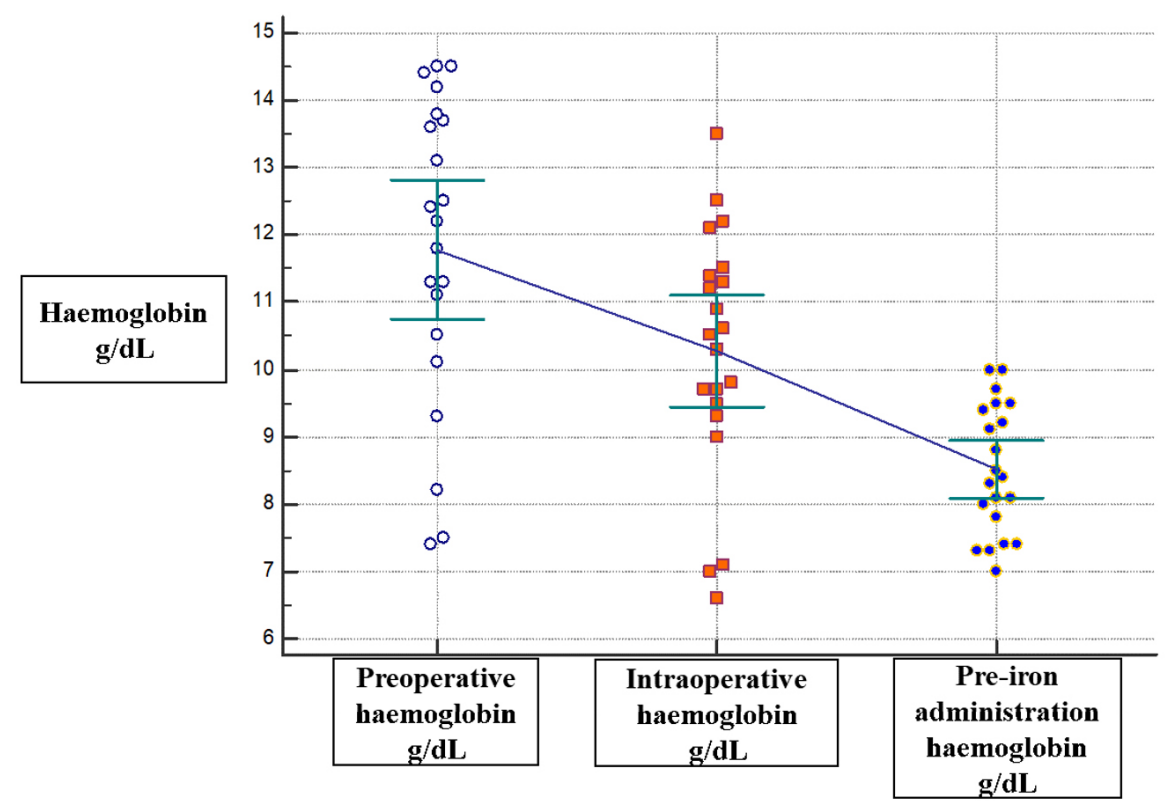

Fig. 3. Haemoglobin levels variation between key moments 
188 The Journal of Critical Care Medicine 2021;7(3)

Table 2. Bioumoral parameters for postoperative iron deficiency screening (mean (SD))

\begin{tabular}{lc} 
Parameters & Values \\
Serum iron $(\mu \mathrm{mol} / \mathrm{L})$ & $3.41(1.67)$ \\
Transferrin saturation $(\%)$ & $7.19(2.74)$ \\
Ferritin $(\mu \mathrm{g} / \mathrm{L})$ & $118.23(17.22)$ \\
$\mathrm{dTIBC}(\mu \mathrm{mol} / \mathrm{L})$ & $48.80(12.20)$ \\
$\mathrm{MCV}(\mathrm{fL})$ & $85.46(6.49)$ \\
$\mathrm{MCH}(\mathrm{g} / \mathrm{dL})$ & $28.34(3.93)$ \\
\hline TSAT = transferrin saturation; dTIBC = direct serum total iron-binding capacity; $\mathrm{MCV}=$ mean
\end{tabular}

corpuscular volume; $\mathrm{MCH}=$ mean corpuscular haemoglobin

Thus, it appears that a high level of glycaemia slows the increase in haemoglobin value (Figure 4). No patient had diabetes nor was treated with insulin. This trend was not encountered for serum lactate, urea or creatinine.

Using Quadratic statistical analysis, even though the mean haemoglobin level between iron administration and post-intervention measurements at 48 and 72 hours did not differ too much $(8.51 \mathrm{~g} / \mathrm{dL}$ versus $8.65 \mathrm{~g} /$ $\mathrm{dL}$ and $8.84 \mathrm{~g} / \mathrm{dL}$ ), the trend analysis indicated $\mathrm{p}$ values $<0.0001$ (Figure 5), meaning that the haemoglobin value is expected to rise over the next period. The multiple trauma patients $(n=5)$ developed acute kidney failure immediately postoperative, but statistical analy-
Available online at: www.jccm.ro

sis showed that it does not influence the haemoglobin correction trend $(\mathrm{p}=0.84)$.

The causal relationship between the length of stay in the hospital and the haemoglobin was analysed, but no correlation was found ( $\mathrm{p}=0.55)$.

In addition, although the included patients presented substantial blood volume loss during the perioperative period, once intravenous iron therapy was initiated, none of the patients required transfusion.

\section{DIsCUSSION}

The term "major surgery" is not standardised yet. However, it is known to include at least abdominal and thoracic surgery, which lasts more than thirty minutes, other extensive surgeries, including neurosurgery and coronary by-pass, significant blood loss (>500 mL), intraoperative use of high vasopressors dose and long intraoperative time $[24,25]$. Therefore, any patient undergoing major surgery is at risk of progressing to anaemia or iron deficiency and in need of haematological optimisation.

Postoperative anaemia and iron deficiency development have many determinants, including blood loss, blood sampling, preoperative anaemia, extensive sur-

Table 3. Postoperative bioumoral parameters measurements. Mean values(SD)

\begin{tabular}{lcc} 
Parameter & $\mathbf{4 8}$ hours after intervention & 72 hours after intervention \\
Glycaemia $(\mathrm{mg} / \mathrm{dL})$ & $125.5(27.59)$ & $124.8(28.28)$ \\
Fibrinogen $(\mathrm{mg} / \mathrm{dL})$ & $227.3(34.07)$ & $250.1(25.15)$ \\
Urea $(\mathrm{mg} / \mathrm{dL})$ & $31.95(6.18)$ & $29.86(5.97)$ \\
Creatinine $(\mathrm{mg} / \mathrm{dL})$ & $1.14(0.30)$ & $1.06(0.22)$ \\
Lactate $(\mathrm{mmol} / \mathrm{L})$ & $1.25(0.48)$ & $1.29(0.34)$ \\
\hline
\end{tabular}
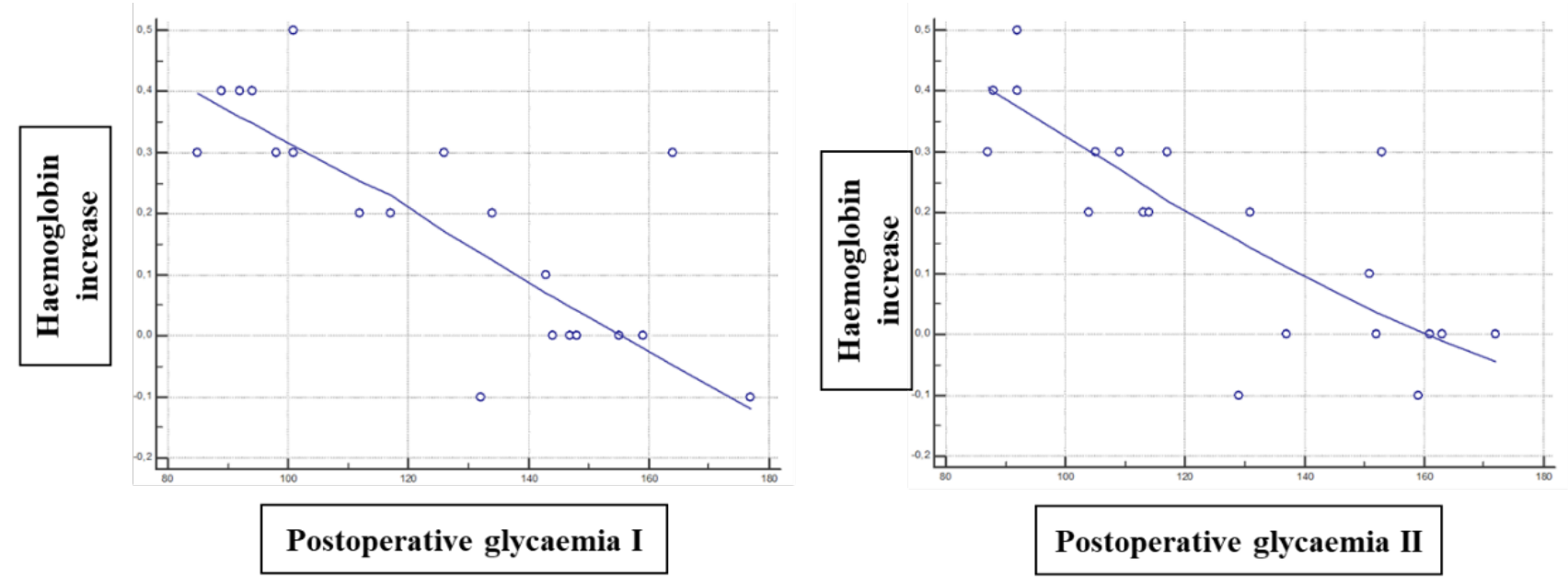

Fig. 4. Glucose influences increase in haemoglobin value 


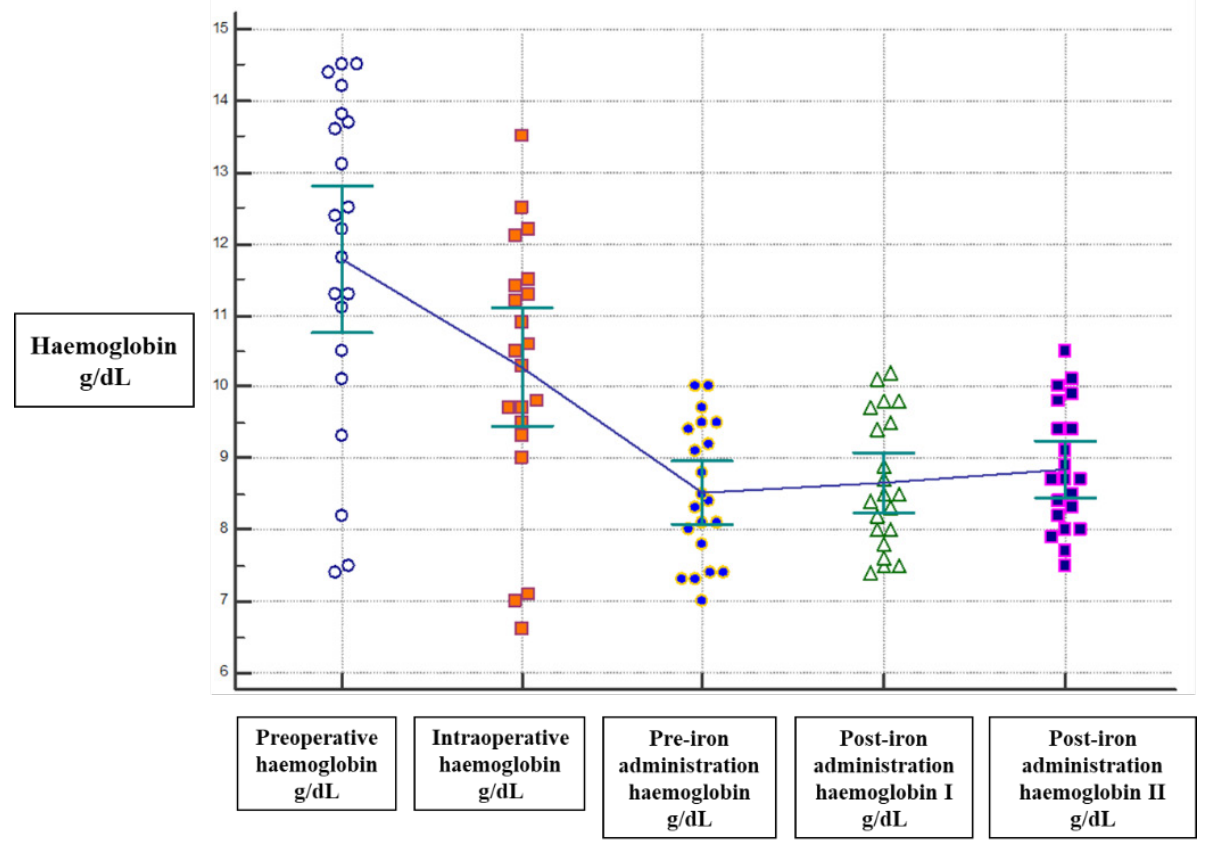

Fig. 5. Quadratic analysis of haemoglobin trend variation

gery and inappropriate nutritional intake related to surgery, and it can appear at any age [26]. The patients in the present study presented either preoperative anaemia or significant intraoperative blood loss, and therefore they developed moderate to severe postoperative anaemia and were screened for iron deficiency. This aspect is more critical in the face of restrictive transfusion strategy (with haemoglobin thresholds depending on various comorbidities), as in our cases.

The importance of iron deficiency screening and treatment in surgical patients arise from the fact that maintaining an absolute iron deficiency may lead to iron deficiency anaemia or iron deficiency without anaemia and specific complications. On the other hand, intravenous iron administration for these patients prevents the appearance of iron-deficiency erythropoiesis $[5,27]$. Additionally, acute kidney injury (AKI) is more often described in patients undergoing major surgical interventions due to different factors, including blood volume waste and iron deficiency. Furthermore, the literature regarding the impact of haemoglobin status and iron deficiency in patients with AKI is inconsistent, and most of the studies analysed the preoperative levels of these deficiencies [12].

Various studies have shown over the years that in the face of a ferritin level $>100 \mu \mathrm{g} / \mathrm{L}$ other markers may be used for iron deficiency screening and diagnosis. Thus, transferrin saturation, serum iron, $\mathrm{MCV}, \mathrm{MCH}$, mean corpuscular haemoglobin concentration (MCHC),
dTIBC, reticulocyte haemoglobin content $(\mathrm{CHr})$, red cell distribution width (RDW) are usually utilised, with soluble transferrin receptor (sTfR) or zinc protoporphyrins (ZPP) in the red cell not measured daily [27-29].

Considering that total daily iron absorption is less than $10 \mathrm{mg} /$ day, the poorer postoperative gastric tolerance and altered gastrointestinal absorption in surgical patients, the use of oral supplements in the postoperative period is inefficient to correct iron deficiency [26, 30]. Various formulas can be used, including ferric carboxymaltose, ferric derisomaltose, iron sucrose, low molecular iron dextran or sodium ferric gluconate. The use of one of these agents is not standardised, being a choice made on availability, experience, patient's allergies, and the available time to correct the iron deficit $[31,32]$.

Some researchers have shown a possible interconnection between iron and glucose metabolism. In healthy subjects, a high intake of glucose appears to increase hepcidin levels, leading to lower values of serum iron [33]. In our patients, high glucose levels were correlated with a decrease in haemoglobin correction trend, maybe due to the same mechanism. In patients with iron deficiency \pm diabetes, high levels of glycated haemoglobin Alc were observed before treatment with iron, which corrected at normal values after the intervention $[34,35]$.

There are still controversies regarding postoperative iron administration. Most studies evaluate preopera- 
tive anaemia/iron deficiency screening and treatment. There are some studies about postoperative intravenous iron agents' use with good outcomes regarding haemoglobin and iron status at 4-weeks follow-up in major elective surgery [36], improved haemoglobin level at two weeks after major orthopaedic surgery [37]. In contrast, some reports found that postoperative iron administration does not significantly correct haemoglobin level or influence the transfusion rate [38]. Therefore, research is needed to clarify the benefits of using postoperative intravenous iron.

\section{CONCLUSION}

At the postoperative screening, all included patients with mean values of intraoperative bleedings more than half a litre presented absolute iron deficiency. Our patients, who received intravenous iron treatment with ferric carboxymaltose, showed a favourable haemoglobin correction trend. Extending the number of patients and the follow-up period will allow us to test our primary hypothesis.

\section{CONFLICT OF INTEREST}

The authors declare no conflict of interest. This study is part of ongoing research for a $\mathrm{PhD}$-thesis.

\section{REFERENCES}

1. WHO. Haemoglobin concentrations for the diagnosis of anaemia and assessment of severity. Vitamin and Mineral Nutrition Information System. Geneva, World Health Organization, 2011 (WHO/NMH/NHD/MNM/11.1); http:// www.who.int/vmnis/indicators/haemoglobin.pdf; accessed April 2021

2. Gómez-Ramirez S, Jericó C, Muñoz M. Perioperative anaemia: Prevalence, consequences and pathophysiology. Transfus Apher Sci. 2019;58:369-74.

3. Steinbicker AU. Role of Anesthesiologists in managing perioperative anemia. Curr Opin Anaesthesiol. 2019;32:64-71.

4. Shander A, Kaufman M, Goodnough LT. How I treat anemia in the perisurgical setting. Blood. 2020;136:814-22.

5. Al-Naseem A, Sallam A, Choudhury S, Thachil J. Iron deficiency without anaemia: a diagnosis that matters. Clin Med (Lond). 2021;21(2):107-13.

6. Camaschella C. Iron deficiency. Blood. 2019;133:30-9.

7. Bodley $T$, Chan M, Clarfield L, et al. Patient Harm from Repetitive Blood Draws and Blood Waste in the ICU: A Retrospective Cohort Study. Blood. 2019;134:57.
8. Auerbach M, Goodnough LT, Shander A. Iron: the new advances in therapy. Best Pract Res Clin Anaesthesiol. 2013;27:131-40.

9. Shander A, Corwin HL. A Narrative Review on HospitalAcquired Anemia: Keeping Blood where It Belongs. Transfus Med Rev. 2020;34:195-9.

10. Andrews NC, Schmidt PJ. Iron homeostasis. Annu Rev Physiol. 2007;69:69-85.

11. Bacuzzi A, Dionigi G, Piffaretti G, et al. Preoperative methods to improve erythropoiesis. Transplant Proc. 2011;43:324-6.

12. Lombardi Y, Ridel C, Touzot M. Anaemia and acute kidney injury: the tip of the iceberg? Clinical Kidney Journal. 2021;14:471-3.

13. Miles LF, Kunz SA, Na LH, Braat S, Burbury K, Story DA. Postoperative outcomes following cardiac surgery in non-anaemic iron-replete and iron-deficient patients - an exploratory study. Anaesthesia. 2018;73:450-8.

14. Miles LF, Larsen T, Bailey MJ, Burbury KL, Story DA, Bellomo R. Borderline anaemia and postoperative outcome in women undergoing major abdominal surgery: a retrospective cohort study. Anaesthesia. 2020;75:210-7.

15. Füllenbach $C$, Stein $P$, Glaser $P$, et al. screening for iron deficiency in surgical patients based on noninvasive zinc protoporphyrin measurements. Transfusion. 2020;60:62-72.

16. Bressman E, Jhang J, McClaskey J, Ginzburg YZ. Tackling the unknowns in understanding and management of hospitalacquired anemia. Blood Rev. 2021.

17. Roubinian NH, Murphy EL, Mark DG, et al. Long-Term Outcomes Among Patients Discharged From the Hospital With Moderate Anemia: A Retrospective Cohort Study. Ann Intern Med. 2019;170:81-9.

18. Makam AN, Nguyen OK, Clark C, Halm EA. Incidence, Predictors, and Outcomes of Hospital-Acquired Anemia. J Hosp Med. 2017;12:317-22.

19. Yamagishi $H$, Okazaki $H$, Shimizu M, Izawa $T$, Komabayashi $T$. Relationships among serum triacylglycerol, fat pad weight, and lipolysis in iron-deficient rats. J Nutr Biochem. 2000 Sep;11(9):455-60.

20. Márquez-Ibarra A, Huerta M, Villalpando-Hernández S, RíosSilva M, Díaz-Reval MI, Cruzblanca H, Mancilla E, TrujilloX. The Effects of Dietary Iron and Capsaicin on Hemoglobin, Blood Glucose, Insulin Tolerance, Cholesterol, and Triglycerides, in Healthy and Diabetic Wistar Rats. PLoS One. 2016 Apr 11;11(4):e0152625.

21. Shu T, Lv Z, Xie Y, Tang J, Mao X. Hepcidin as a key iron regulator mediates glucotoxicity-induced pancreatic $\beta$-cell dysfunction. Endocr Connect. 2019 Mar 1;8(3):150-161.

22. Finch CA, Gollnick PD, Hlastala MP, Miller LR, Dillmann E, Mackler B. Lactic acidosis as a result of iron deficiency. J Clin Invest. 1979 Jul;64(1):129-37.

23. https://www.mdcalc.com/ganzoni-equation-iron-deficiencyanemia, accessed May 2020.

24. Martin D, Mantziari S, Demartines N, Hübner M; ESA Study 
Available online at: www.jccm.ro

Group. Defining Major Surgery: A Delphi Consensus Among European Surgical Association (ESA) Members. World J Surg. 2020;44:2211-9.

25. Anderson FA Jr, Spencer FA. Risk factors for venous thromboembolism. Circulation. 2003;107:19-16.

26. Muñoz M, Franchini M, Liumbruno GM. The postoperative management of anaemia: more efforts are needed. Blood Transfus. 2018;16:324-5.

27. Richards T, Breymann C, Brookes MJ, et al. Questions and answers on iron deficiency treatment selection and the use of intravenous iron in routine clinical practice. Ann Med. 2021;53(1):274-85.

28. Wish JB. Assessing iron status: beyond serum ferritin and transferrin saturation. Clin J Am Soc Nephrol. 2006;1:S4-8.

29. Kang CK, Pope M, Lang CC, Kalra PR. Iron deficiency in heart failure: Efficacy and safety of intravenous iron therapy. Cardiovasc Ther. 2017;35.

30. Elstrott B, Khan L, Olson S, Raghunathan V, DeLoughery T, Shatzel JJ. The role of iron repletion in adult iron deficiency anemia and other diseases. Eur J Haematol. 2020;104:153-61.

31. Muñoz M, Gómez-Ramírez S, Bhandari S. The safety of available treatment options for iron-deficiency anemia. Expert Opin Drug Saf. 2018;17:149-59.

32. https://www.accessdata.fda.gov/drugsatfda_docs/ label/2020/208171s000lbl.pdf; accessed May 2021.

33. Aigner E, Felder TK, Oberkofler H, Hahne P, Auer S, Soyal S,
The Journal of Critical Care Medicine 2021;7(3) • 191

Stadlmayr A, Schwenoha K, Pirich C, Hengster P, Datz C, Patsch $W$. Glucose acts as a regulator of serum iron by increasing serum hepcidin concentrations. J Nutr Biochem. 2013 Jan;24(1):112-7. doi: 10.1016/j.jnutbio.2012.02.017. [Epub 2012 Jul 21].

34. Kim C, Bullard KM, Herman WH, Beckles GL. Association between iron deficiency and $\mathrm{A} 1 \mathrm{C}$ Levels among adults without diabetes in the National Health and Nutrition Examination Survey, 1999-2006. Diabetes Care. 2010 Apr;33(4):780-5.

35. Bhardwaj K, Sharma SK, Rajpa N, Sachdev A. The effect of iron deficiency anaemia on haemoglobin A1c levels. Ann Clin Lab Res. 2016 Oct;4(4):123.

36. Khalafallah AA, Yan C, Al-Badri R, et al. Intravenous ferric carboxymaltose versus standard care in the management of postoperative anaemia: a prospective, open-label, randomised controlled trial. Lancet Haematol. 2016;3:e415-25.

37. Kim MS, Koh IJ, Choi KY, Yang SC, In Y. Efficacy and Safety of Intravenous Ferric Carboxymaltose in Patients with Postoperative Anemia Following Same-Day Bilateral Total Knee Arthroplasty: A Randomized Controlled Trial. J Clin Med. 2021;10:1457.

38. Jeong JH, Chang MJ, Kang SB, Park HJ, Lee KH, Chang CB. Postoperative Intravenous Iron Supplementation Does Not Improve Haemoglobin Level, and Transfusion Rate Following Staged Bilateral Total Knee Arthroplasty. J Arthroplasty. 2020;35:2444-50. 